\title{
Research on Intelligent Control of Pipe Dredging Robot
}

\author{
Xiaomei Jiang ${ }^{1, a}$, Yannian Rui ${ }^{2, b}$ \\ ${ }^{1}$ School of Mechanical Engineering, Changshu Institute of Technology Changshu, 215500, China \\ ${ }^{2}$ College of Mechatronics Engineering, Soochow University, Suzhou, 215021, China \\ ajszjjxm@hotmail.com, ${ }^{b}$ ryn@suda.edu.cn
}

Keywords: dredging robot; adaptive control; extension transformation; compliance control

\begin{abstract}
Drainpipe transports the production waste water and life waste water in modern urban infrastructure, due to its small branch pipe diameter, it is easy to foul, if not desilting in time will result in overflow of sewage, which not only seriously affect city's appearance, but also cause environmental pollution. The operating environment in underground drainpipe is usually more extreme and complicated or that human can not adapt to an environment, therefore, drainpipe desilting is an extremely important and heavy work. Many problems of pipe dredging robot on adaptive control for complex situation need to be solved. In this paper, based on the analysis of traditional intelligent control methods and comparison of principle of adaptive control and extension control, a new intelligent method - "extension and adaptive control" was established combining adaptive control with extension control. Extension and adaptive controller and compliance control planning are formed and used in the control system of drainpipe dredging robot, improves its level of intelligence, offers the theoretical foundation for the application of this control method. Results show this method has better adaptability to environment than the traditional adaptive control in driven control of drainpipe dredging robot.
\end{abstract}

\section{Introduction}

Drainpipe is one of the main underground pipes in modern urban infrastructure, for transporting the production waste water and life waste water. Due to small branch pipe diameter, it is easy to foul, if not desilting in time will result in overflow of sewage, which not only seriously affect city's appearance, but also cause environmental pollution[1]. Therefore, drainpipe desilting is an extremely important and heavy work. The operating environment in underground drainpipe is usually more extreme and complicated or that human can not adapt to an environment, drainpipe dredging robot can complete certain job functions and solve problems like quality inspection, maintenance, fault diagnosis and pipeline operation of most pipes unable to be approached, avoid low efficiency and not guaranteed safety. With the continuous advancement of intelligent control and sensor technology, using a robot to fulfill certain job tasks in such a harsh environment became human choice. Research on walking style, operating pose, desilting tool, vehicle power, detection method and control method has considerable difficulty, combined with working space constraint, makes it hard to select and install necessary sensor. Because the drainpipe deposition is very complex, often unpredictable obstacles for robot, so very easy to tilt and deflection, deviating from the center to the wall, damage cutting tool and the wall, the robot can't work normally[2].

Due to the complexity of the environment, a lot of problems in adaptive control of robot need to be solved. Traditional adaptive control is good at dealing with constant and slow time-varying, but can not solve the unpredictable, fast time-varying problems or unexpected events in process. For example, when drainpipe conditions are good, adaptive controller can automatically adjust strategy according to direction, speed to make the robot progress stably. However, when drainpipe situation is complex, conditions beyond the definite strategic scope, adaptive driving control can’t do anything [3].

Although in recent years, intelligent control method like artificial neural networks, fuzzy control can simulate the human brain wisdom, but there is obvious lag for complex systems with unexpected events. The expert system's knowledge base and inference engine can be used to reason decision-making problems and get the relevant conclusions. However, the majority of knowledge 
systems and expert systems have the surface knowledge. To solve complex problems, storage, representation and processing of deep knowledge must be addressed in the knowledge-based systems to improve problem-solving capacity and flexibility [4].

The extension control is characterized by good performance in meeting an emergency, and uses a formal model to study the extensive possibility to expand and develop innovative rules and methods and deal with conflicts. Extension model provide a combination of qualitative and quantitative model for the artificial intelligence expression [5]. This paper attempts to construct extension and adaptive controller in drainpipe dredging robot. Adaptive control is used when the environment remains unchanged or little change, and extension control when environmental change.

\section{Extension Adaptive Control System Design}

Basic principle of extension and adaptive control of pipe dredging robot is shown in Fig.1.

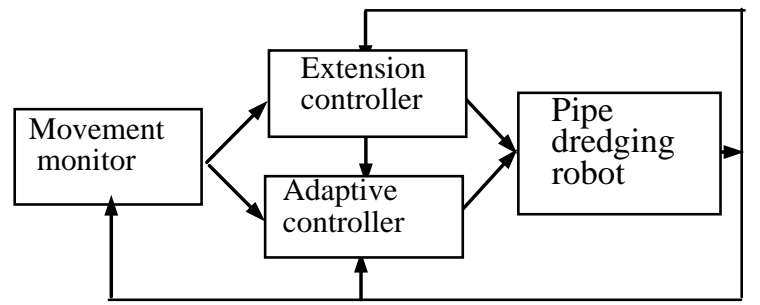

Fig .1 Extension adaptive control block diagram

There are three running state in control system: EC separately, EC and AC together, AC separately, monitor MM is responsible for switching between them. The structure of extension controller is shown in Fig.2. Among them general object is a equivalent including actuator, the controlled object and detection device, the remaining five modules are the key elements of extension controller.

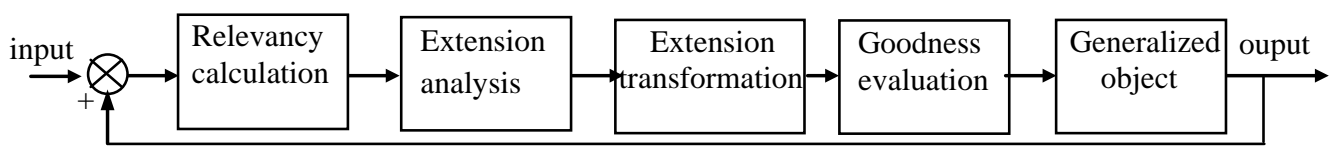

Fig. 2 The structure of extension controller

Design of Extension Controller. Establish extension model of control: the key control issues mainly contain control objectives and control conditions, and denote control objectives and control conditions as the primitive element (including the matter element, event element and relationship element). In pipeline dredging robot control, assumed control objectives and control conditions being: $R=\left[\begin{array}{ccc}\text { pipe dredging robot } A, & \text { velocity } c_{1}, & v_{1} \\ & \begin{array}{c}\text { direction } c_{2}, \\ \text { expected deviation }\end{array} c_{3}, & v_{2}\end{array}\right]=\left[\begin{array}{ccc}A, & c_{1} & v_{1} \\ c_{2} & v_{2} \\ c_{3} & v_{3}\end{array}\right]$

$r_{1}=\left[\begin{array}{cccc}\text { pipe dredging robot } A, & \text { dirt thickness } & c_{4} & v_{4} \\ & \text { slight dirty } & c_{5} & \\ & v_{5} \\ & \text { actual deviation } & c_{6} & v_{6}\end{array}\right]=\left[\begin{array}{ccc}A, & c_{4} & v_{4} \\ & c_{5} & v_{5} \\ & c_{6} & v_{6}\end{array}\right]$

$r_{2}=\left[\begin{array}{cccc}\text { operating condition } B, & \text { pipe conditions } c_{4} & v_{4} \\ & \text { tilt degree } c_{5} & v_{5} \\ & \text { outside interferen ce } c_{6} & v_{6}\end{array}\right]=\left[\begin{array}{ccc}A, & c_{7} & v_{7} \\ c_{8} & v_{8} \\ c_{9} & v_{9}\end{array}\right]$

Among them, $r_{1} \wedge r_{2}$, that is, control problem is to implement $R$ at condition $r$, denoted by: $P=R * r$. From the feature model control, then the set of characteristic state is:

$S=\left(c_{1}, c_{2}, c_{3}, c_{4}, c_{5}, c_{6}, c_{7}, c_{8}, c_{9}\right)$ 
Relevancy calculation: establish extension set according to the core control issue and calculate relevancy in three cases:

(1) $G(p) \geqslant 0$, Control is compatible, then the control can achieve the desired control objectives by the conventional adaptive control method, this phase is the adaptive control stage.

(2) $-1 \leqslant G(p) \leqslant 0$, Control is incompatible, if only the conventional adaptive control methods can not turn incompatibility into compatibility. But by adding some auxiliary means of extension control, the incompatibility control can translate into compatibility, then the conventional adaptive control can achieve desirable control, i.e. "adaptive control + extension control "stage.

(3) $G(p) \leqslant-1$,Control is incompatible, and adaptive control completely ineffective. This stage was divided into two cases: extension control turn incompatibility into compatibility; and opposite.

Adaptive control of robots is closely related to dynamics. The structure of self-correction adaptive controller is shown in Fig.3. The structure of the adaptive controller based on extension set is shown in Fig.4. Consider the single-variable system, assume ${ }^{x}$ be set value of the system, ${ }^{y}$ be output of the generalized object. The system error $\delta=x-y$, error change rate $\dot{\delta}=d e / d t$. According to empirical data, determine the output variables be classical domain element and knot domain element of $\delta$ and $\dot{\delta}$.

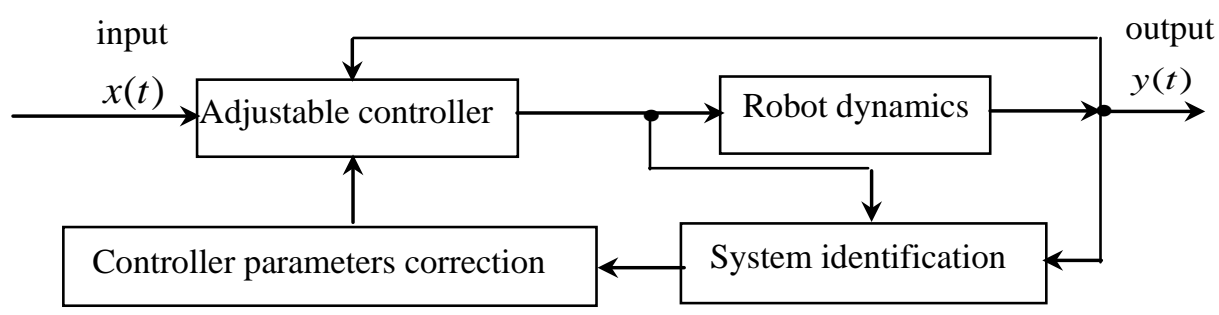

Fig. 3 Self-correction adaptive controller

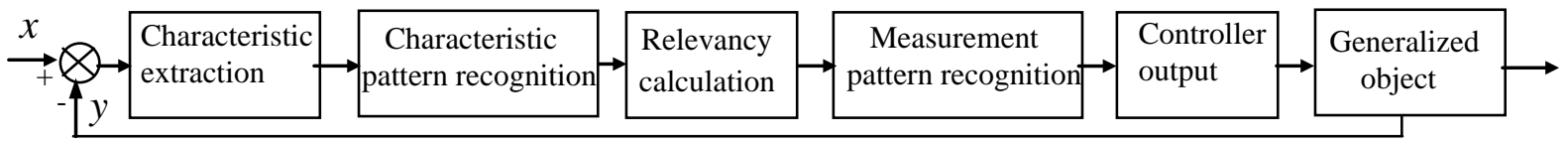

Fig. 4 The structure of adaptive extension controller

\section{Compliance control planning}

In many cases, force or torque control of the robot and position control are equally important. When the actuator of the robot contact with surrounding environment, only the position control are often unable to meet the requirements[6]. In this issue, robot works in the underground, and the environment is not the same, and the foul density is not uniform, and moisture content are not consistent, so in this case it is a torque control. When the foul is rather hard, withstanding torque is large, the robot speed should slow down, and in places the quality of the soft foul or loose, torque is small, the robot speed should be appropriately as quick as possible. So, productivity is significantly improved, robot construction can be very well protected. We adopt an improved $\mathrm{R}$ - C controller, Fig.5 is the R - C hybrid control system structure.

In Fig. 5, $S$ and $\bar{S}$ are fit selection array, $x_{d}$ and $F_{d}$ are desired position and force track in the definition of the Cartesian coordinate system, $P(q)$ is the robot kinematic equation, ${ }^{c} T$ is force transformation array.

On the basis of R-C controller, in view of the dynamic coupling influence not considered in controller, this can cause the robot to appear not stably certain non-exotic location in working space, make the following improvement: in hybrid controller, taking into account the dynamic effects, compensate the gravity and the Coriolf's force and the centripetal force suffered; considering the under-damping characteristic in torque control system, in the control loop, join the damping feedback, so as to weaken oscillating factors; Acceleration feedforward is introduced to meet the job task requirements for acceleration, speed can be a smooth transition. 


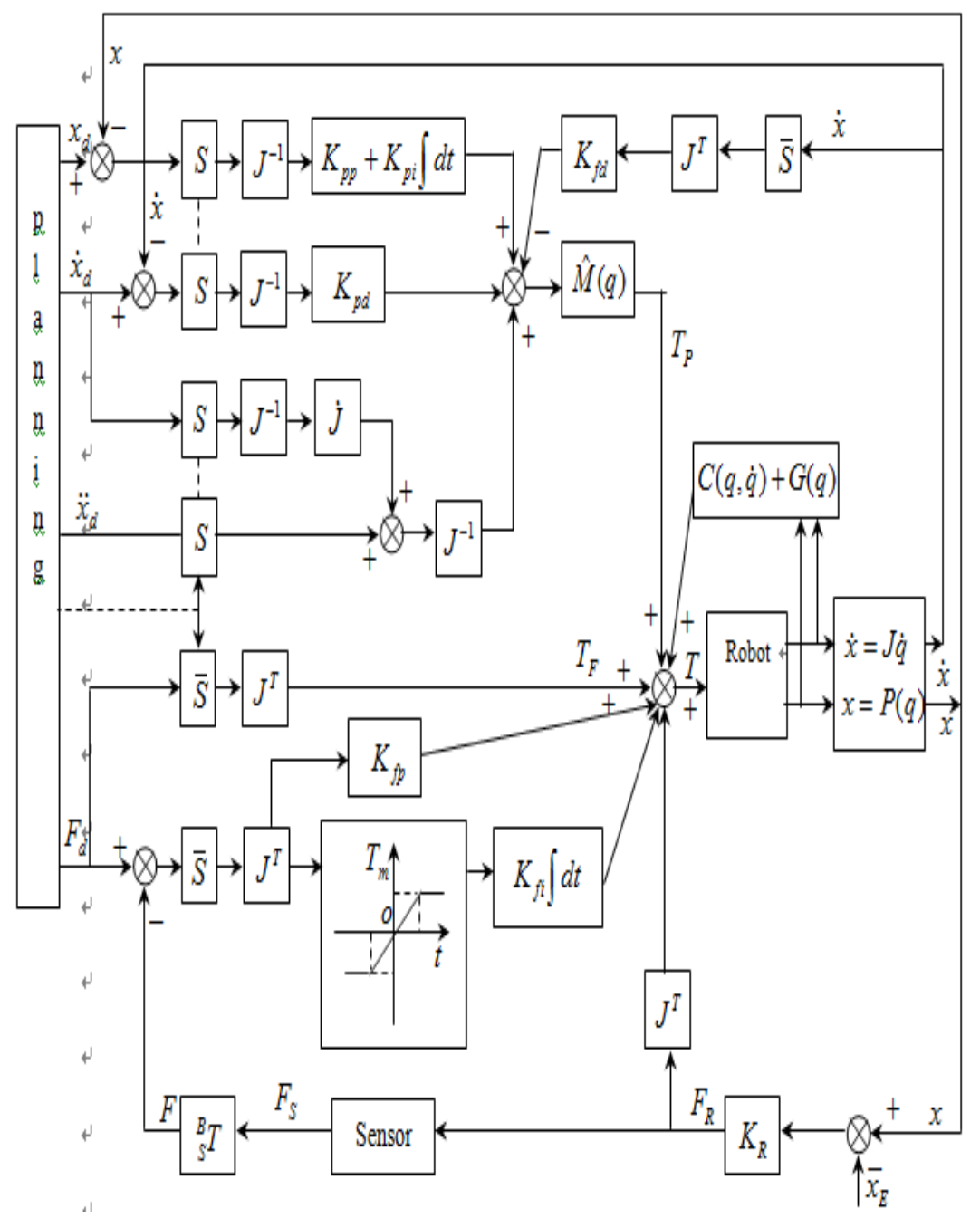

Fig .5 R-C structure of hybrid control system

\section{Summary}

According to the above analysis, this paper presents a new type of intelligent control system design of pipe dredging robot which can combine the advantages of both adaptive control and extension control. The adaptive extension control method and compliance control planning are formed and used in the control system of drainpipe dredging robot, improves its level of intelligence, offers the theoretical foundation for the application of this control method.

\section{References}

[1] J. Wang, J. Liu and G. Song, The new removal method of the urban sewer sediment, Water Sciences and Engineering Technology, (2009) 46-48.

[2] P.C. Ji, H.P. Shen, J.M. Deng, W. Zhu and J.G. Xu, A new sewer desilting robot control system design, China Rural Water and Hydropower, (2010) 64-67.

[3] Z.X. Cai, Intelligent control theory and application, Tsinghua University Press, Beijing (2007).

[4] D.C. Yuan, Modern Control Theory, Tsinghua University Press, Beijing (2007).

[5] C.Y. Yang and W. Cai, Extension Engineering, Science Press, Beijing (2007).

[6] F. Wang and G. Zhang, Autonomous Drainpipe Dredging Robot Based on Fuzzy Control, Machine tool \& Hydrulics, 42 (2009) 41-43. 